\title{
Diagnóstico y alternativas terapéuticas en la isquemia miocárdica aguda perioperatoria en cirugía coronaria
}

\author{
A.M. Bueno-González ${ }^{a}, *$, J.L. Pérez-Vela ${ }^{a}$, F. Hernández ${ }^{b}$, E. Renes ${ }^{a}$, P. Arribas ${ }^{a}$, \\ M.A. Corres ${ }^{a}$, J. Gutiérrez ${ }^{a}$ y N. Perales ${ }^{a}$ \\ aUnidad de Postoperatorio Cardiológico, Servicio de Medicina Intensiva, Hospital Universitario 12 de Octubre, \\ Madrid, España \\ ' Unidad de Hemodinámica y Cardiología Intervencionista, Servicio de Cardiología, Hospital Universitario 12 de Octubre, \\ Madrid, España
}

Recibido el 22 de septiembre de 2008; aceptado el 29 de noviembre de 2008

Disponible en Internet el 6 de octubre de 2009

\section{PALABRAS CLAVE \\ Infarto \\ perioperatorio; \\ Cirugía de \\ revascularización; \\ Isquemia miocárdica; \\ Biomarcadores; \\ Angiografía; \\ Intervención \\ coronaria percutánea}

\section{KEYWORDS}

Perioperative

myocardial infarction;

Myocardial

revascularization

surgery;

\begin{abstract}
Resumen
La isquemia y el infarto posrevascularización quirúrgica son unas complicaciones relativamente frecuentes y pueden conllevar una morbimortalidad elevada. El diagnóstico precoz es fundamental, pero está menos estandarizado y es más complicado que en los pacientes que no reciben cirugía cardíaca, y no existe ningún marcador específico capaz de distinguir entre la isquemia miocárdica en relación con el procedimiento en sí y el daño miocárdico asociado a infarto perioperatorio. Ante la detección de isquemia perioperatoria, se debe proceder de inmediato para instaurar medidas con la intención de limitar el daño miocárdico.

Los objetivos de este estudio son los siguientes: 1) revisar los métodos diagnósticos disponibles para la isquemia miocárdica aguda perioperatoria y los criterios de infarto agudo de miocardio perioperatorio; 2) revisar las diferentes opciones terapéuticas existentes, y 3) proponer un algoritmo de tratamiento que contempla la necesidad de realizar un diagnóstico diferencial, el control del vasoespasmo, la implantación de balón de contrapulsación y las posibles estrategias de revascularización (intervención coronaria percutánea o reintervención quirúrgica).

(c) 2008 Elsevier España, S.L. y SEMICYUC. Todos los derechos reservados.
\end{abstract}

Diagnostic and therapeutic alternatives in perioperative acute myocardial ischemia in heart surgery

\section{Abstract}

Ischemia and infarct after surgical revascularization are a relatively frequent complication, with high morbidity and mortality. Early diagnosis is essential. However, this is less

\footnotetext{
*Autor para correspondencia.

Correo electrónico: ana_buegon@yahoo.es (A.M. Bueno-González).
} 
Myocardial ischemia; Myocardial injury markers; Angiography;

Percutaneous coronary intervention standardized and more complicated to diagnose than in patients who have not undergone surgery since there is no specific biomarker that allows the clinician to differentiate between myocardial ischemia due to the procedure itself and myocardial damage due to perioperative infarct.

Once detected, perioperative ischemia should be treated immediately in order to limit myocardial damage.

The objectives of this study have been 1. To show the diagnostic criteria for perioperative infarct and ischemia. 2. to show the different therapeutic options available. 3. to propose a treatment algorithm that includes the differential diagnosis, how to control vasospasm, implantation of balloon counterpulsation, and the possible revascularization strategies (percutaneous coronary intervention vs reoperation).

(c) 2008 Elsevier España, S.L. and SEMICYUC. All rights reserved.

\section{Introducción}

A pesar de los continuos avances en las técnicas quirúrgicas, en los métodos de protección miocárdica y en el tratamiento perioperatorio y postoperatorio, el infarto agudo de miocardio perioperatorio (IAMp) posrevascularización quirúrgica se observa con una incidencia entre el 5 y el $10 \%{ }^{1}$, y depende fundamentalmente de los criterios diagnósticos utilizados. No disponemos de un estándar de referencia diagnóstico de IAMp fácilmente aplicable a la cabecera del enfermo. De hecho, la elevación de marcadores de daño miocárdico, así como las alteraciones electrocardiográficas, son hallazgos frecuentes en el postoperatorio de cirugía cardíaca no complicada ${ }^{2}$. Se admite que algún grado de isquemia miocárdica transitoria es inevitable, y ocurre en más del $40 \%$ de los pacientes, la mayoría en las primeras horas de postoperatorio. Además, ningún marcador es capaz de distinguir entre isquemia miocárdica en relación con el procedimiento en sí y el daño miocárdico asociado a IAMp ${ }^{3,4}$.

Aunque el perfil de los pacientes está cambiando, con una tendencia cada vez mayor a intervenir pacientes de mayor edad, con cirugía de revascularización previa, con enfermedad coronaria más extensa, con mayor comorbilidad y peor función ventricular, la incidencia del IAMp no ha cambiado de manera significativa, probablemente debido a los avances en los métodos de preservación miocárdica, en las técnicas quirúrgicas (incluyendo el uso de injertos arteriales) y en el desarrollo de la monitorización intraoperatoria y postoperatoria.

A pesar de ello, esta complicación conduce a un aumento del consumo de los recursos sanitarios ${ }^{5}$ y a un incremento en la morbimortalidad hospitalaria ${ }^{4,6}$.

La isquemia que aparece tras la revascularización quirúrgica puede ser el resultado de espasmo arterial, oclusión o estenosis del injerto o del árbol nativo por trombosis aguda o en relación con la anastomosis. El control del espasmo, la mejoría de la perfusión coronaria mediante contrapulsación o la consecución de una revascularización adecuada mediante intervención coronaria percutánea (ICP), reintervención o injerto adicional podrían salvar el miocardio y proporcionar un beneficio clínico a corto y a largo plazo de los pacientes ${ }^{7-9}$.

Cabe destacar la escasez de información en la literatura médica sobre cómo detectar a estos enfermos y las pautas terapéuticas por seguir. Con esta premisa que dificulta la toma de decisiones, el objetivo de este trabajo pretende revisar qué criterios precoces de isquemia postoperatoria podrían utilizarse y qué pauta de tratamiento terapéutico podría establecerse para intentar limitar el daño miocárdico permanente tras una revascularización quirúrgica. Concretamente se evalúa el papel potencial de la angiografía coronaria y el intervencionismo posterior en pacientes seleccionados.

\section{Factores de riesgo y predictores de isquemia miocárdica aguda perioperatoria}

La identificación de factores predictores es importante para entender la patogenia del daño perioperatorio y para el desarrollo de estrategias preventivas ${ }^{10,11}$.

Los factores de riesgo asociados a isquemia son múltiples y pueden estar en relación con condiciones preoperatorias o intraoperatorias $^{1,6}$. En el análisis multivariante de un estudio prospectivo realizado en 499 pacientes, Greaves et $\mathrm{al}^{12}$ identificaron la cirugía de urgencia, el tiempo de pinzamiento aórtico (TCAo) superior a $100 \mathrm{~min}$, el infarto en la semana previa y la revascularización previa como factores independientes asociados a un incremento de IAMp. En otro estudio, Chaitman et al $^{13}$ encontraron una mayor incidencia de IAMp en pacientes con cardiomegalia, tiempos operatorios prolongados (tiempo de by-pass cardiopulmonar [BCP] superior a $180 \mathrm{~min}$ y TCAo superior a $100 \mathrm{~min}$ ), reintervención y revascularización combinada con otra cirugía cardíaca.

También se han descrito las concentraciones plasmáticas de troponina $(\mathrm{Tn})$ I preoperatoria como predictoras de episodios cardíacos adversos y mortalidad intrahospitalaria $^{14,15}$. En un reciente estudio prospectivo realizado en 776 pacientes, Onorati et al $^{10}$ mostraron los siguientes como factores de riesgo independientes: EuroSCORE superior a 6 (IC: 4,91-6,62; $p=0,002$ ), angina inestable con cambios en el ECG (IC: $1,04-1,54 ; p=0,03$ ), TCAo superior a $90 \mathrm{~min}$ (IC: $2,03-2,58 ; p=0,02$ ), tiempo de BCP superior a $180 \mathrm{~min}$ (IC: $5,92-8,71 ; \quad p=0,001)$, revascularización incompleta (IC: $501-7,19 ; p=0,001)$ y balón de contrapulsación intraaórtico (BCIAo) intraoperatorio (IC: 2,68-4,59; $\mathrm{p}=0,001)$.

Actualmente, los ensayos epidemiológicos genéticos estudian la asociación de determinados polimorfismos genéticos en la respuesta inflamatoria asociada al BCP con 
un mayor riesgo de IAMp. Así, en una reciente publicación ${ }^{16}$ se asocian diversas variantes genéticas en los patrones de liberación de citocinas y de interacción leucocito-endotelio asociada al BCP como factores independientes asociados a la gravedad de la mionecrosis después de la cirugía cardíaca. La identificación de éstos puede permitir la identificación preoperatoria de pacientes de alto riesgo y el desarrollo de nuevas estrategias cardioprotectoras ${ }^{1,17}$.

\section{Etiología de isquemia miocárdica aguda perioperatoria}

Cabe destacar que, tras cirugía de revascularización, algún grado de isquemia miocárdica transitoria ocurre en aproximadamente el $40 \%$ de los pacientes. Esto se debe al propio procedimiento quirúrgico (cardiotomía, canulación, cardioplejía y fenómenos embólicos en relación con el daño endotelial), que suponen discretos y transitorios aumentos de biomarcadores, fundamentalmente en las primeras horas después de la cirugía ${ }^{17}$. Un $3-10 \%$ de los pacientes presenta una lesión isquémica clínicamente manifiesta, con alteraciones en el ECG, inestabilidad hemodinámica o arritmias ventriculares.

Son múltiples los factores y las causas de isquemia perioperatoria, que se esquematizan en la tabla $1^{4,12,18-21}$. Una causa frecuente y potencialmente reversible de isquemia perioperatoria es el vasoespasmo coronario, que puede afectar a las arterias coronarias sanas, a los vasos nativos con injertos y a los injertos arteriales, principalmente descritos en los de la arteria radial, pero también producidos en los de mamaria interna.

\section{Patogénesis y tratamiento de vasoespasmo coronario}

La etiología es incierta y se han descrito múltiples factores precipitantes que afectan tanto al período intraoperatorio como al período postoperatorio; durante la cirugía la propia manipulación y la lesión endotelial pueden inducir vasoespasmo, y en el postoperatorio el uso de fármacos vasoconstrictores, la hipotermia, el aumento del tono alfaadrenérgico, la liberación del tromboxano A2 plaquetario como rebote al retirar fármacos bloqueantes de los canales del calcio o la hipomagnesemia de alta incidencia en el postoperatorio son algunos de los factores propuestos. La utilización de injertos arteriales y la mejoría de los resultados clínicos a largo plazo, con el menor desarrollo de aterotrombosis y la mayor durabilidad de los injertos, ha promovido el desarrollo de la cirugía coronaria arterial, pero tiene inconvenientes. El más importante es el mayor poder de vasorreactividad de las arterias, aunque no todas tienen el mismo potencial espástico; la arteria radial es la de mayor riesgo ${ }^{22}$. Por esto, se establece la necesidad de utilizar de forma sistemática y protocolizada la profilaxis antivasoespástica en estos pacientes de alto riesgo. El diagnóstico clínico de vasoespasmo puede ser difícil de hacer; generalmente se manifiesta por la elevación del segmento ST en múltiples derivaciones, el colapso hemodinámico o las arritmias ventriculares. El tratamiento del vasoespasmo incluye optimizar la oxigenación, corregir la acidosis y los factores precipitantes y aportar nitroglicerina o un bloqueante de los canales del calcio. La coronariografía permite la confirmación del diagnóstico y su resolución con la administración de fármacos vasodilatadores locales en pacientes que por el deterioro cardiovascular que presentan no tolerarían su administración sistémica ${ }^{18,23-25}$.

\section{Diagnóstico de isquemia miocárdica aguda perioperatoria}

Se ha descrito una elevación de biomarcadores sobre el límite superior al normal en el $62-90 \%$ de los pacientes intervenidos ${ }^{1,4,26}$, lo que supone un grado de necrosis miocárdica que se considera inevitable en relación con el procedimiento. Asimismo, los cambios en el ECG y las alteraciones de la contractilidad por ecocardiografía se pueden encontrar en otras situaciones frecuentes en un postoperatorio inmediato normal. A continuación analizamos los diferentes elementos diagnósticos de isquemia aguda perioperatoria.

1. Elevación de biomarcadores: si se entiende el aumento de los biomarcadores como inherente al propio acto quirúrgico, y queremos utilizarlos para diagnóstico de infarto perioperatorio, es fundamental determinar un punto de corte (tanto de creatincinasa MB masa [CPK] como de troponina $\mathrm{T}[\mathrm{TnT}]$ y troponina $\mathrm{I}[\mathrm{Tnl}]$ ) que tenga la sensibilidad y la especificidad suficientes ${ }^{27-33}$.

Se acepta que el biomarcador de mayor sensibilidad y especificidad disponible hoy para el diagnóstico de IAMp tras cirugía de revascularización es la $\mathrm{TnI}^{34}$. El pico normal tras la cirugía sin complicaciones se obtiene en torno a las $12 \mathrm{~h}$ y decrece a las $24 \mathrm{~h}$; en caso de daño miocárdico establecido

Tabla 1 Etiología en la isquemia miocárdica perioperatoria ${ }^{4,7,12,18-21}$

\begin{tabular}{|c|c|c|}
\hline Injerto/árbol nativo & Cirugía & $\begin{array}{l}\text { Aumento de la demanda/ } \\
\text { disminución de flujo }\end{array}$ \\
\hline $\begin{array}{l}\text { 1. Espasmo de las arterias coronarias o de los injertos } \\
\text { 2. Trombosis aguda de la arteria nativa o del injerto venoso (más } \\
\text { frecuente) } \\
\text { 3. Acodamiento y estiramiento de los injertos } \\
\text { 4. Estenosis de anastomosis/complicaciones técnicas (más } \\
\text { frecuente injertos arteriales) }\end{array}$ & $\begin{array}{l}\text { 1. Microembolización } \\
\text { aterotrombótica } \\
\text { 2. Embolización aérea } \\
\text { 3. Protección subóptima }\end{array}$ & $\begin{array}{l}\text { 1. Fallo de bomba e hipotensión } \\
\text { 2. Hipertensión y taquicardia } \\
\text { 3. Fenómeno de robo de la subclavia }\end{array}$ \\
\hline
\end{tabular}

1. Espasmo de las arterias coronarias o de los injertos

frecuente)

3. Acodamiento y estir frecuente injertos arteriales) 
Table 2 Troponinas

\begin{tabular}{|c|c|c|}
\hline Autor, año y referencia & Resultados & Comentarios \\
\hline Tzimas et al, $2008^{34}$ & $\begin{array}{l}\text { - } \mathrm{Tnl}>13,5 \mu \mathrm{g} / \mathrm{l} \text { a las } 12 \mathrm{~h} \text { post-Cx } \\
\text { - } \mathrm{Tnl}>13,4 \mu \mathrm{g} / \mathrm{l} \text { a las } 24 \mathrm{~h} \text { post-Cx }\end{array}$ & $\begin{array}{l}\text { E: } 81 \% \text { S: } 60 \% \\
\text { Sistema de análisis: INMULITE } \\
2000 \mathrm{TnI}\end{array}$ \\
\hline Nahum et al, $2008^{35}$ & $\begin{array}{l}\text { - } \mathrm{TnT}>0,8 \mu \mathrm{g} / \mathrm{l}(8 \text { veces el límite superior } \\
\text { normal) } \\
\text { - } \mathrm{TnT}>1,3 \mu \mathrm{g} / \mathrm{l}\end{array}$ & $\begin{array}{l}\text { Predictor independiente de } \\
\text { episodios cardíacos en cirugía } \\
\text { aislada (coronaria o valvular) } \\
\text { Predictor de episodios cardíacos } \\
\text { después de cirugía combinada }\end{array}$ \\
\hline Noora et al, $2005^{36}$ & $\begin{array}{l}\text { - La Tnl media en pacientes con IAMp fue } \\
\text { de } 46,85 \mu \mathrm{g} / \mathrm{l} \text {. } \\
\text { - Tnl }>2,3 \mu \mathrm{g} / \mathrm{l} \text { diagnóstico de IAMp por el } \\
\text { sistema Abbot AxSym System }\end{array}$ & $\begin{array}{l}\text { Sistema de análisis: Sistema } \\
\text { Abbot AxSym System }\end{array}$ \\
\hline & - $\mathrm{Tnl}>15 \mu \mathrm{g} / \mathrm{l}$ a las $30-48 \mathrm{~h}$ post-Cx & S: $100 \% . \mathrm{E}: 91 \%$ \\
\hline Thielman et al, 2004. Eur J Card Surg ${ }^{37}$ & $\begin{array}{l}\text { - Tnl: } 21,5 \mu \mathrm{g} / \mathrm{l} \text { a las } 12 \mathrm{~h} \\
\text { - Tnl: } 33,4 \mu \mathrm{g} / \mathrm{l} \text { a las } 24 \mathrm{~h}\end{array}$ & $\begin{array}{l}\text { S: } 82 \% . \text { E: } 66 \% \\
\text { S: } 98 \% . \text { E: } 82 \% \\
\text { Análisis: Dimension Flex }{ }^{\circledR}\end{array}$ \\
\hline Onorati et al, 2005. Ann Torca Surg. ${ }^{10}$ & - $\mathrm{Tnl}>3,1 \mu \mathrm{g} / \mathrm{l}$ a las $12 \mathrm{~h}$ post-Cx & Access Immunoassay System \\
\hline Carrier et al, 2000. Ann Torca Surg. ${ }^{30}$ & $\begin{array}{l}\text { - } \mathrm{Tnl}>3,9 \mu \mathrm{g} / \mathrm{l} \text { a las } 24 \mathrm{~h} \text { post-Cx } \\
\text { - } \mathrm{TnT}>3,4 \mu \mathrm{g} / \mathrm{l} \text { a las } 28 \mathrm{~h}\end{array}$ & $\begin{array}{l}\text { S: } 80 \% . \text { E: } 85 \% \\
\text { S: } 90 \% . \text { E: } 94 \% \\
\text { Baxter Stratus Analyzer }\end{array}$ \\
\hline $\begin{array}{l}\text { Castro Martínez et al, 2002. Rev Esp } \\
\text { cardiol }^{40}\end{array}$ & - $\mathrm{Tnl}>12 \mu \mathrm{g} / \mathrm{l}$ & S: $91 \%$. E: $89 \%$ \\
\hline & & $\begin{array}{l}\text { Inmunoanálisis con anticuerpos } \\
\text { monoclonales (no especifica) }\end{array}$ \\
\hline Peivandi et al, $2004^{33}$ & $\begin{array}{l}\text { - TnT: } 3 \mu \mathrm{g} / \mathrm{l} \text { a las } 120 \mathrm{~h} \text {. IC del } 95 \% \\
\text { - Tnl: } 70,5 \mu \mathrm{g} / \mathrm{l} \text { a las } 24 \mathrm{~h} \text {. IC del } 95 \%\end{array}$ & \\
\hline Januzzi et al, 2002. J Am Coll Card. ${ }^{20}$ & - $\mathrm{TnT}>1,58 \mu \mathrm{g} / \mathrm{l}$ a las $18-24 \mathrm{~h}$ & \\
\hline Holmvang et al, $2002^{6}$ & - $\mathrm{TnT}>1,75 \mu \mathrm{g} / \mathrm{l}$ & S: $71 \% . \mathrm{E}: 68 \%$ \\
\hline Fransen et al, 2002. Chext. ${ }^{32}$ & $\begin{array}{l}\text { - } \mathrm{TnT}>1 \mu \mathrm{g} / \mathrm{l} \text { a las } 8 \mathrm{~h} \\
\text { - S: } 76,9 \% . \mathrm{E}: 72,7 \%\end{array}$ & \\
\hline Benoit et al, 2001. Critical Care Med. ${ }^{31}$ & $\begin{array}{l}\text { - } \mathrm{Tnl}>19 \mu \mathrm{g} / \mathrm{l} \text { a las } 12 \mathrm{~h} \\
\text { - } \mathrm{Tnl}<36 \mu \mathrm{g} / \mathrm{l} \text { a las } 24 \mathrm{~h}\end{array}$ & $\begin{array}{l}\text { S: } 100 \% . \text { E: } 73 \% \\
\text { S: } 100 \% . \text { E: } 93 \% \\
\text { Stratus II DATE Behring. }\end{array}$ \\
\hline $\begin{array}{l}\text { Alyanakian et al, 1998. Cardiothorac Vasc } \\
\text { Anest. }{ }^{38}\end{array}$ & $\begin{array}{l}-\mathrm{Tnl}<15 \mu \mathrm{g} / \mathrm{l} \text { entre } 24-48 \mathrm{~h} \text { post-Cx } \\
\text { excluye el diagnóstico de IAMp. }\end{array}$ & Análisis: Stratus ${ }^{\circledR}$ \\
\hline Gensini et al, 1998. Crit Care Med. ${ }^{39}$ & - $\mathrm{Tnl}>9,2 \mathrm{mcg} / \mathrm{l}$ a las $12-24 \mathrm{~h}$ & $\begin{array}{l}\text { S: } 100 \% . \text { E: } 100 \% \\
\text { Stratus II DADE International }\end{array}$ \\
\hline
\end{tabular}

Valores diagnósticos y predictores de IAMp.

Cx: cirugía; E: especificidad; IAMp: infarto agudo de miocardio perioperatorio; IC: intervalo de confianza; S: sensibilidad; Tn: troponina.

la cinética de liberación es diferente, con un pico más tardío y más prolongado en el tiempo. Son múltiples los estudios que intentan establecer el punto de corte más sensible y específico para el diagnóstico de IAMp, como se refleja en la tabla $2^{6,32,34-41}$. Para su interpretación, hay que considerar, además, que se han comercializado varios ensayos para medir Tnl, cuyos valores varían según el modelo/hardware utilizado en cada laboratorio (Opus Plus o Abbot AxSym immunoassay), y explica así la amplia variabilidad de valores en los diferentes estudios. En el estudio de Noora et $\mathrm{al}^{36}$ un valor de $\mathrm{Tnl}$ superior a $2,3 \mu \mathrm{g} / \mathrm{l}$ se consideró diagnóstico de IAMp mediante el sistema de Abbot AxSym. En comparación, los ensayos de análisis para las isoformas de TnT tienen sólo un modelo, esto corrobora la mayor validez externa de los resultados y la mayor consistencia entre laboratorios, pero está menos disponible y es más caro $^{36,37,42}$

En un estudio reciente, Nahum et $\mathrm{al}^{35}$ correlacionaron los niveles de TnT con los episodios cardíacos perioperatorios, incluido el IAMp, en función del tipo de cirugía (coronaria, valvular o cirugía combinada). Las elevaciones sobre el valor normal $(0,1 \mu \mathrm{g} / \mathrm{l})$ se observaron en el $90 \%$ de los pacientes a las $24 \mathrm{~h}$ poscirugía. Sin embargo, una elevación de TnT 8 veces superior al límite normal $(0,8 \mu \mathrm{g} / \mathrm{l})$ fue un predictor independiente de episodios cardíacos después de cirugía coronaria o cirugía valvular aislada. Un valor superior a $1,3 \mu \mathrm{g} / \mathrm{l}$ se encontró predictor de episodios cardíacos después de cirugía combinada de alto riesgo.

En el documento de consenso publicado por la American College of Cardiology/American Heart Association 
(ACC/AHA) en el año $2007^{43}$ para la redefinición universal del IAM, la elevación de biomarcadores se considera criterio diagnóstico de IAMP tras cirugía de revascularización coronaria (CRC) cuando los valores son superiores a 5 veces el percentil 99 del rango de referencia normal en las primeras $72 \mathrm{~h}$ tras la cirugía 44 .

En este consenso se define el IAMp en relación con la CRC cuando esta elevación de biomarcadores se asocia a la aparición de nuevas ondas $\mathrm{Q}$, nuevo bloqueo de rama izquierda (LBBB) u oclusión angiográfica de los injertos o evidencia por imagen de pérdida del miocardio viable ${ }^{43}$.

2. Cambios electrocardiográficos: las manifestaciones del ECG de isquemia se producen precozmente, de manera que pueden usarse para discriminar a los pacientes con isquemia miocárdica aguda perioperatoria.

2.A. Cambios en el segmento ST: las alteraciones del segmento ST son indicativas de isquemia y predictoras de IAMp en un número no despreciable de pacientes, pero también se pueden presentar en el postoperatorio precoz, de forma transitoria, en otros enfermos que no asocian isquemia aguda. Tal es el caso de pacientes en hipotermia, con pericarditis, cambio en la posición de los electrodos, pacientes con repolarización precoz, bloqueos de rama, alteraciones iónicas o vasoespasmo ${ }^{45}$. Además, la existencia de LBBB completo, la estimulación ventricular y otros trastornos frecuentes tras el despinzamiento aórtico pueden hacer difícil su valoración ${ }^{46}$.

2.B. Nuevas ondas Q: aparecen en el 4-5\% de los pacientes tras CRC y, aunque normalmente reflejan necrosis miocárdica aguda, también se han descrito excepcionalmente al desenmascarar un IAM previo ${ }^{4}$. Además, se ha señalado en la literatura médica algún caso con ondas $Q$ transitorias durante la cirugía, que se atribuyen a diferentes causas, como isquemia o hipoxia, espasmo coronario, trastornos metabólicos, trastornos electrolíticos o hipoter$\mathrm{mia}^{1}$. Salvo estas excepciones, poco frecuentes, una nueva onda $\mathrm{Q}$ debe considerarse diagnóstica de infarto (empeora el pronóstico y aumenta la mortalidad a corto plazo) ${ }^{13}$.

No está claro el valor del LBBB de nueva aparición en relación con isquemia tras la cirugía, si bien la última revisión ACC/AHA del 2007 la incluye como criterio diagnóstico en asociación a otros ${ }^{43}$.

3. Ecocardiografía transesofágica (ETE): la ETE, capaz de demostrar nuevas alteraciones de la contractilidad segmentaria, es también de utilidad para el diagnóstico de IAMp, si bien su interpretación puede ser complicada dado que en el postoperatorio inmediato la contractilidad miocárdica global está deprimida en un número alto de pacientes, fundamentalmente en relación con aturdimiento miocárdico ${ }^{1,47}$.

\section{Criterios diagnósticos de isquemia e infarto agudo de miocardio perioperatorio}

La sintomatología clínica habitual de angina, que se observa en la cardiopatía isquémica, no suele ser útil para el diagnóstico tras cirugía cardíaca. Así, el diagnóstico de IAMp se establece sobre la base de criterios del ECG (nuevas ondas Q, alteraciones del segmento ST o LBBB), la liberación de biomarcadores y las alteraciones segmentarias de la contractilidad por ecocardiografía, y considera a la coronariografía como el estándar de referencia diagnósti$\mathrm{co}^{6,18,19,45,48}$.

A nivel práctico, para poder tomar actitudes de tratamiento en los pacientes tras CRC, podemos considerar como criterios diagnósticos los siguientes:

A. Sospecha de isquemia perioperatoria aguda: la detección de uno o más de estos criterios puede realizarse desde el ingreso del paciente y puede conllevar actitudes diagnósticas o terapéuticas:

1. Cambios del ECG en el segmento ST, definidos como elevación o depresión del segmento ST superior a $2 \mathrm{~mm}$ en 2 derivaciones consecutivas comparado con el ECG preoperatorio.

2. Episodios repetidos de taquicardia ventricular (TV) o fibrilación ventricular (FV).

3. Deterioro hemodinámico, no atribuible a otras causas, con signos de fracaso ventricular izquierdo con descenso de gasto cardíaco (GC) e incremento de presión auricular izquierda después de incrementar soporte inotrópico o balón de contrapulsación intraaórtico (BCIAo).

4. Alteraciones graves en la contractilidad miocárdica en la misma área que las alteraciones del segmento ST o acinesia o hipocinesia en comparación con el ecocardiograma preoperatorio.

5. LBBB.

B. Diagnóstico de IAMp: establece el diagnóstico de infarto y supone daño miocárdico establecido, pero requiere el paso del tiempo para confirmar los hallazgos y no conduce a la realización de maniobras terapéuticas.

1. Desarrollo de nuevas ondas $Q$ en el ECG, definidas como significativas si son superiores a 0,04 ms en 2 derivaciones, o reducción en ondas $\mathrm{R}$ mayor del $25 \%$ en al menos 2 derivaciones.

2. Según la AHA/ACC, elevación de CPK-MB superior a 5 veces el límite normal superior, en las primeras $72 \mathrm{~h}$ tras la CRC; en general, valor de CPK-MB superior a $125 \mathrm{U} / \mathrm{l}$. Las elevaciones de Tn superiores a 5 veces el límite superior al normal. Para la isoforma de Tnl, es difícil establecer un punto de corte de referencia, ya que varían según las técnicas de inmunoanálisis de cada laboratorio, lo que hace difícil su comparación.

\section{Pronóstico de infarto agudo de miocardio perioperatorio}

El IAMp es un episodio clínico que afecta negativamente al pronóstico. Aunque es ampliamente aceptado que el IAMp aumenta la morbimortalidad intrahospitalaria en los primeros 30 días, con una alta incidencia de episodios cardíacos, no ocurre así con el pronóstico a largo plazo, donde hay mayores discrepancias sobre la repercusión pronósti$\mathrm{ca}^{9,49,50}$.

Con la intención de estratificar y analizar el pronóstico de los pacientes que han presentado un IAM tras CRC, se desarrolló un estudio observacional ${ }^{51}$, en el que se analizó a 59 pacientes que habían presentado un IAMp y a 115 pacientes sin IAMp, y se estudió el desarrollo de episodios cardíacos a los 30 meses (muerte, infarto, angina inestable e 
insuficiencia cardíaca). Los pacientes con IAMp tuvieron mayor incidencia de episodios cardíacos (el 31 versus el $12 \%$, $\mathrm{p}<0,01$ ), y la mayoría ocurrieron en los 3 primeros meses. En el análisis de regresión de Cox se identificaron 2 factores predictores independientes de episodios cardíacos además de IAMp (riesgo relativo [RR]: 2,7), la revascularización incompleta (RR: 3,5$)$ y una FE postoperatoria del ventrículo izquierdo inferior al 40\% (RR: 2,1).

En un estudio prospectivo, realizado en 776 pacientes, Onorati et $\mathrm{al}^{10}$ evaluaron el pronóstico de los pacientes con criterios de ECG, ecocardiográficos y bioquímicos para IAMp en aquellos que presentaban únicamente elevación de concentraciones plasmáticas de Tnl (lo que denominan "daño miocárdico" perioperatorio). Se estudió la supervivencia inicial y a los 12 meses y la ausencia o no de episodios cardiovasculares. Así, los pacientes con valores de concentraciones plasmáticas de Tnl superiores a 3,1 ng/l a las $12 \mathrm{~h}$ se detectaron en el 6,9\% (54 de los 776 pacientes analizados), y se asociaron a una menor supervivencia intrahospitalaria $(p<0,001)$, a una menor supervivencia al alta $(p=0,00001)$ y a una mayor incidencia de episodios cardíacos $(p=0,0009)$. En los pacientes que además presentaron criterios del ECG y ecocardiográficos de IAMp (21 de los 54 pacientes) se observaron valores más altos de concentraciones plasmáticas de Tnl y se asociaron a una incidencia más alta de episodios cardiovasculares $(\mathrm{p}=0,0084)$, a tiempos más prolongados de ventilación mecánica ( $p=0,001)$, a mayor mortalidad intrahospitalaria $(p=0,003)$ y a mayor mortalidad al alta $(p=0,023)$. El valor pronóstico de la TnT quedó también de manifiesto en un análisis multivariante, en el que las concentraciones de TnT superiores o iguales a $58 \mathrm{ng} / \mathrm{ml}$ (representan 5 veces el límite superior) fueron un fuerte predictor de muerte postoperatoria o shock en el inmediato postoperatorio o de las 18 a las $24 \mathrm{~h}^{9}$. También se demostró una clara relación entre el grado de elevación de la CPK-MB, el pronóstico y la tasa de mortalidad a largo plazo. En una revisión de 2.918 pacientes ${ }^{18,51}$, la mortalidad a los 6 meses, asociada a elevaciones de CPK-MB $<5, \geq 5 \mathrm{a} \leq 10, \geq 10 \mathrm{a} \leq 20, \mathrm{y} \geq 20$ veces el límite superior de la normalidad, fue del 3,4 , el 5,8 , el 7,8 , y el $20,2 \%$, respectivamente. Esta relación permaneció significativa después de ajustar por otros factores de riesgo.

En cuanto a los cambios del ECG, el desarrollo de una nueva onda $Q$ ha mostrado ser fuertemente indicativa de peor pronóstico en el CASS (Coronary Artery Surgery Study $)^{1,4,13}$. De esta manera, la mortalidad de los pacientes con nueva onda $Q$ fue del 10 frente al $1 \%$ en los que no la desarrollaron. Cabe destacar que en este estudio no se encontraron diferencias entre ambos grupos en la mortalidad a largo plazo (el $5 \%$ a los 3 años). No se ha encontrado asociación de la mortalidad a otros nuevos cambios del ECG (elevación o depresión del segmento ST o inversión de onda $T)^{13,18}$.

\section{Tratamiento: indicaciones de angiografía y estrategias de revascularización}

Los datos disponibles en la literatura médica sobre cómo detectar y tratar a los pacientes con isquemia miocárdica temprana en el postoperatorio inmediato de revascularización coronaria son sorprendentemente limitados. En un intento de establecer el tratamiento y los criterios de reangiografia en los pacientes con isquemia aguda perioperatoria, se realizó una búsqueda bibliográfica, y se destacaron los siguientes estudios. Rasmussen et $\mathrm{al}^{47}$ analizaron a 71 pacientes con sospecha de fallo del injerto (el $3,5 \%$ de los 2.003 pacientes que recibieron revascularización), y los dividieron en 2 grupos según se derivaran a reangiografía (grupo $1, n=59$ ) o a reoperación inmediata (grupo 2, $n=12$ ), en función de la estabilidad hemodinámica. En el grupo 1 se demostró fallo del injerto o revascularización incompleta en 43 pacientes (73\%). Basados en los hallazgos de la coronariografía, 27 pacientes se reoperaron y se reinjertaron, y los otros 16 pacientes se trataron de forma conservadora. La mortalidad a los 30 días fue del $7 \%$. En el grupo 2, en el que se realizó reoperación inmediata, sin angiografía, 11 pacientes (92\%) mostraron fallo del injerto y la mortalidad a los 30 días en éstos fue del $50 \%$. Los autores concluyen que, en un elevado porcentaje de los pacientes con criterios de isquemia precoz y estabilidad hemodinámica, la realización de una coronariografía proporciona hallazgos relevantes (fallo del injerto o revascularización incompleta), lo que condiciona un diagnóstico y un tratamiento quirúrgico ulteriores.

Fabricius et $\mathrm{al}^{48}$ estudiaron una cohorte de 131 pacientes con isquemia miocárdica perioperatoria (el 6,4\% de los 2.052 pacientes que recibieron revascularización); de ellos, a 108 se les realizó reangiografía, mientras que a 23 se los reoperó inmediatamente debido al deterioro hemodinámico grave. La angiografía mostró problemas relacionados con el injerto o la revascularización incompleta en 63 pacientes. De éstos, 43 pacientes se reintervinieron (34 pacientes mediante nueva cirugía y 9 pacientes mediante ICP), mientras que los otros 20 pacientes no se reintervinieron debido a los malos lechos distales mostrados en la angiografía. La mortalidad a los 30 días fue del $9,3 \%$ en el grupo en que se realizó angiografía y del $39 \%$ en el grupo de revascularización quirúrgica inmediata, sin angiografía previa. Se confirmó infarto definitivo (elevación de CPK-MB superior al 10\%, cambios significativos en el segmento ST o nueva onda $Q$ ) en 72 pacientes $(57,4 \%)$ del grupo de angiografía y en 21 pacientes $(91,3 \%)$ del grupo con reintervención quirúrgica inmediata.

En estos 2 estudios se observó que la realización de una angiografía en pacientes con estabilidad hemodinámica y criterios de isquemia aguda puede ayudar a definir a los pacientes que se benefician de reintervención o ICP. A diferencia, los pacientes inestables hemodinámicamente y aquéllos con malos lechos distales presentan malos resultados globales, con una mayor mortalidad ${ }^{47,48}$. En estos pacientes es más complejo definir pautas de actuación, y obliga a individualizar en cada caso el riesgo/beneficio de las intervenciones.

Thielmann et $\mathrm{al}^{15}$, para intentar definir la mejor estrategia de revascularización en los pacientes con isquemia aguda perioperatoria, realizaron un estudio angiográfico a 118 pacientes de los 5.427 intervenidos quirúrgicamente para revascularización miocárdica. La coronariografía mostró fallo del injerto en 67 pacientes; de éstos, a 25 se les realizó ICP, a 15 se los reintervino y a los otros 27 se les hizo tratamiento conservador. En los pacientes que recibieron ICP se encontró un menor ascenso de biomarcadores que en el grupo reintervenido quirúrgicamente, pero no encontraron diferencias significativas, ni en la función ventricular, ni en la mortalidad cuando se 
Tabla 3 Tratamiento perioperatorio y estrategias de revascularización en los estudios más relevantes

\begin{tabular}{llll}
\hline & Rasmussen et al $^{47}$ & Fabricius et al $^{48}$ & Thielmann et al $^{15}$ \\
\hline $\begin{array}{l}\text { Reangiografia, pacientes } \\
\text { Hallazgos/fallo del injerto, } \mathrm{n}(\%)\end{array}$ & 59 & 108 & 118 \\
$\quad$ Revascularizados & $43(72)$ & $63(58)$ & $67(56)$ \\
$\quad$ Cirugía & 27 & 43 & 40 \\
$\quad$ ICP & 16 & 34 & 15 \\
$\quad$ Tratamiento conservador & 12 & 9 & 25 \\
Cirugía inmediata & $11(92)$ & 23 & 27 \\
Hallazgos/fallo del injerto, n (\%) & $19(82)$ & 27 \\
\hline
\end{tabular}

ICP: intervención coronaria percutánea.

compararon los grupos. En el análisis de evaluación de resultados postintervención, ambos grupos obtuvieron buenos resultados. Por tanto, este estudio no mostró una clara diferencia entre una u otra opción de revascularización, y subrayó la necesidad de una investigación conjunta para determinar el mejor tratamiento de fallo de injerto tras cirugía de revascularización, dado que sigue siendo un tema, cuanto menos, controvertido. Los resultados de estos 3 estudios se resumen en la tabla 3.

Una técnica de gran utilidad para el diagnóstico intraoperatorio de complicaciones con las anastomosis o con los injertos es la medición del flujo intracoronario por flujimetría Doppler. Tiene la ventaja de identificar errores técnicos (estenosis, rotación, acodamientos de los injertos, etc.) mientras el esternón está abierto. Se trata de una medida mínimamente invasiva que podría realizarse en todo paciente revascularizado $y$, sobre todo, en aquéllos con signos de fallo del injerto durante la cirugía, como es el caso de pacientes con elevación intraoperatoria persistente del segmento ST. Algunos autores están a favor de su uso habitual como método preventivo. Los flujos intracoronarios medios superiores a $15 \mathrm{ml} / \mathrm{min}$, los índices de pulsatilidad entre 1-5 y las curvas de flujo de predominio diastólico descartarían inicialmente problemas técnicos con las anastomosis y con los injertos ${ }^{21,52-55}$. Más recientemente se ha introducido la angiografía intraoperatoria (fluorescent indocyanine green), que proporciona imágenes angiográficas similares a la angiografía- $R x^{56,57}$. La identificación intraoperatoria de un fallo en el injerto permite la revisión inmediata y el control de éste o la realización de un injerto adicional.

A la vista de la literatura médica, la sospecha de fallo temprano del injerto requiere intervención inmediata; tras tratamiento del vasoespasmo y valoración de contrapulsación, la estrategia recomendable es realizar angiografía a los pacientes estables con sospecha de isquemia miocárdica aguda. A diferencia, en los pacientes con importante inestabilidad hemodinámica o clínica se debe intentar la estabilización, la implantación de BCIAo y posteriormente plantear la angiografía. La angiografía identifica la causa de fallo del injerto y es diagnóstica y terapéutica de vasoespasmo, y permite la administración de vasodilatadores locales. En los que por su situación no puede realizarse coronariografía, una vez descartadas otras causas de inestabilidad, habría que evaluar la posibilidad de revascularización quirúrgica directa? ${ }^{9}$ No obstante, estos
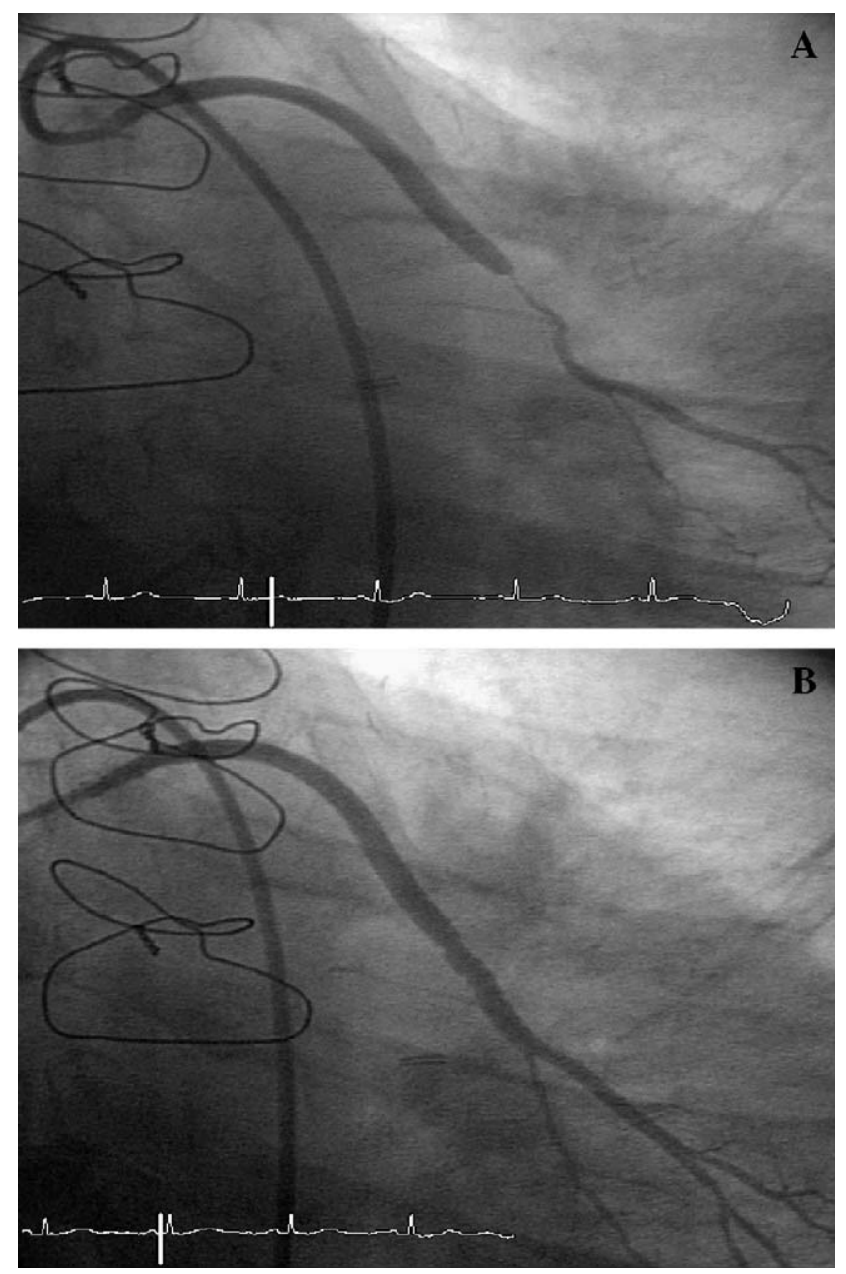

Figura 1 Injerto aortocoronario de la vena safena a obtusa marginal en proyección oblicua anterior derecha. A) Lesión crítica en la anastomosis distal. B) Resultado angiográfico tras angioplastia e implante de stent farmacoactivo.

pacientes asocian una mayor mortalidad, probablemente relacionada con la deteriorada situación hemodinámica ${ }^{19}$.

En la práctica clínica habitual, la estrategia de revascularización óptima para la oclusión/fallo del injerto no está clara y no hay un tratamiento estandarizado para estos pacientes $^{4,6,30,48}$. En la mayor parte de las situaciones, las 
decisiones se toman de una manera consensuada, y se trata cada caso concreto de una forma individualizada. En general, las técnicas de intervencionismo coronario se consideran menos invasivas $y$, siempre que sean factibles por el tipo y la localización de la lesión, algunos autores las consideran como la primera opción de tratamiento, con lo que consiguen altos porcentajes de revascularización (fig. 1). Esta alternativa es particularmente útil cuando se encuentran los problemas en el árbol nativo y en los pacientes hemodinámicamente estables. Según las recomendaciones de ACC/AHA del 2005 en "Guideline update for coronary artery bypass graft surgery", el ICP en pacientes con CRC puede considerarse como el tratamiento de elección en la isquemia temprana posrevascularización quirúrgica $^{2,58}$.

La reoperación para revascularización es una opción por considerar tanto en pacientes estables como en pacientes inestables $y$, fundamentalmente, en aquéllos con evidencia de revascularización incompleta, problemas de anastomosis o con el injerto una vez valorado el riesgo/beneficio y las opciones posibles de revascularización ${ }^{19,21,31,48}$.

Criterios de sospecha de isquemia miocardica aguda:

- Nuevos cambios ECG en el segmento ST, definidos como elevación o depresión más de 2 mm en dos derivaciones consecutivas, no descritas en ECG previos.

- Deterioro hemodinámico, no atribuible a otras causas como sangrado, con signos de fracaso ventricular a pesar de soporte circulatorio.

- Episodios repetidos de FV-TV.

- Alteraciones severas de la contractilidad segmentaria miocárdica (ecocardio) concordantes con los cambios ECG.

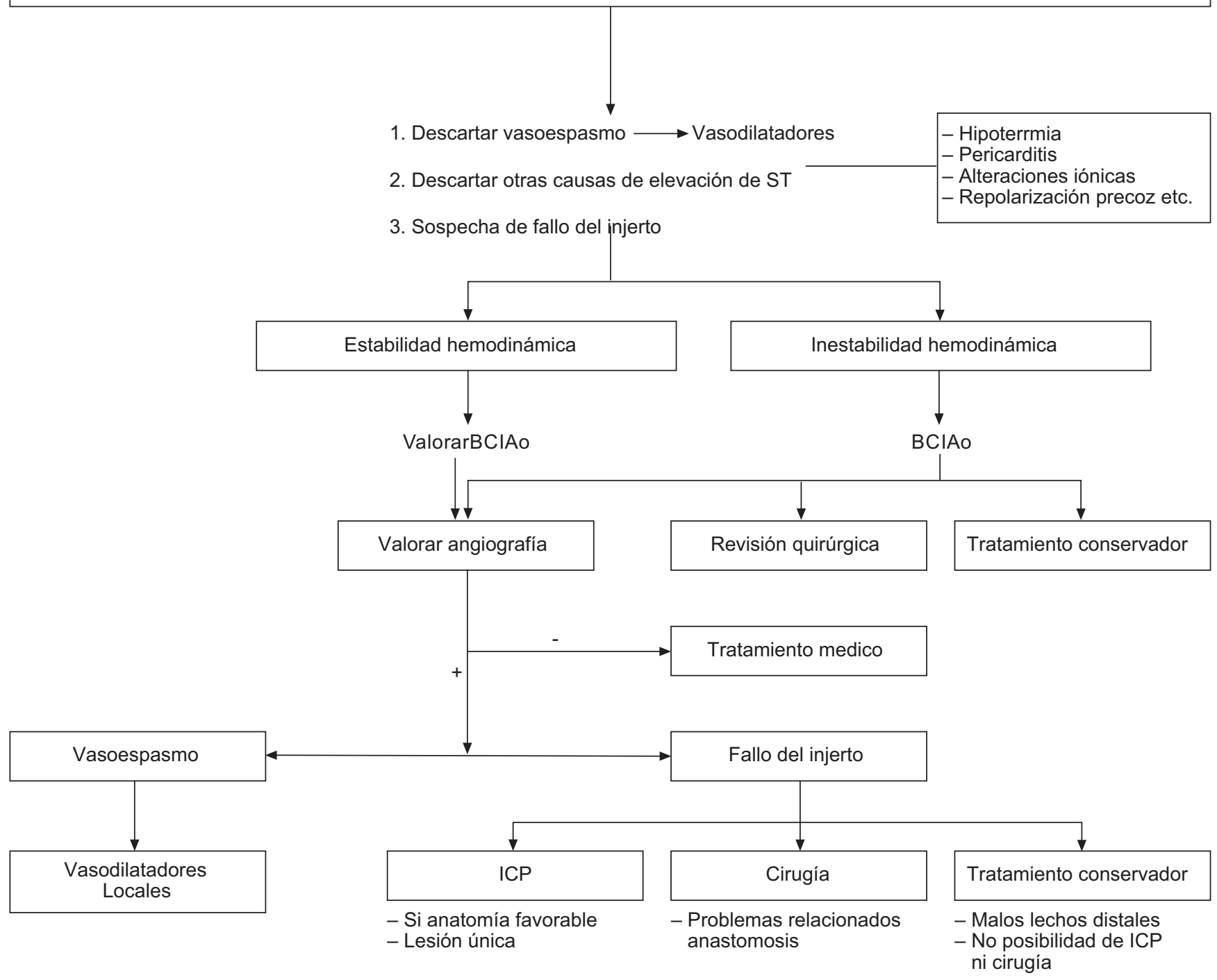

BCIAo: Balón de contrapulsación intra-aórtico

ICP: Intervención Coronaria Percutánea.

Figura 2 Algoritmo de actitud diagnóstico-terapéutica y tratamiento. 
En el caso de pacientes con malos lechos coronarios, sin posibilidad de ICP, sólo se puede plantear un tratamiento médico conservador con medidas antiisquemia, antitrombóticas, uso de vasodilatadores si se sospecha vasoespasmo y soporte circulatorio con BClAo en caso de inestabilidad hemodinámica.

\section{Conclusiones}

El diagnóstico precoz de la isquemia miocárdica aguda perioperatoria es fundamental, y es más complicado que en los pacientes que no reciben cirugía cardíaca, dado que son múltiples las causas que pueden alterar los marcadores diagnósticos de necrosis en este contexto. Así, en el postoperatorio, ante la sospecha de isquemia miocárdica aguda y, una vez planteado un diagnóstico diferencial, se debe intervenir de inmediato para limitar el daño miocárdico, ya sea con tratamiento vasodilatador (en caso de sospecha de vasoespasmo) o con estrategias de revascularización en caso de fallo o problemas con los injertos. Esta revisión pone en evidencia la falta de consenso tanto en los criterios diagnósticos como en la actitud más adecuada para estos pacientes. Por esto, son necesarios estudios clínicos multicéntricos y multidisciplinarios que incluyan a intensivistas, a cirujanos cardíacos y a cardiólogos intervencionistas para clarificar las estrategias de tratamiento más adecuado para estos pacientes. Sobre la base de esta revisión, se propone un algoritmo de tratamiento para estas situaciones (fig. 2).

\section{Bibliografía}

1. Jain U. Myocardial infarction during coronary artery bypass surgery. J Cardiothorac Vasc Anesth. 1992;6:612-23.

2. Eagle KA, Guyton RA, Davidoff R, F. Edwards, G. Ewy, T. Gardner, et al. ACC/AHA 2004 guideline update for coronary artery bypass graft surgery: A report of the American College of Cardiology/American Heart Association Task Force Practice Guidelines. Circulation. 2004;110:e340.

3. Califf RM, Abdelmeguid AE, Kuntz RE, Pompa JJ, Davidson CJ, Cohen EA, et al. Myonecrosis after revascularization procedures. J Am Coll Cardiol. 1998;31:241-51.

4. Uptodate. Early complications of coronary artery bypass graft surgery [citado 5 Sep 2009]. Disponible en: URL: http:// uptodate.papi.h12o.es/online/content/topic.do?topi cKey=chd $/ 59270 \&$ selectedTitle=2 150\&source=search_result.

5. Chen JC, Kaul P, Levy JH, Haverich A, Menasché P, Smith PK, et al. Myocardial infarction following coronary artery bypass graft surgery increases healthcare resource utilization. Crit Care Med. 2007;35:1296-301.

6. Holmvang L, Jurlander B, Rasmussen C, Thiis J, Grande P, Clemmensen $P$. Use of biochemical markers of infraction for diagnosing perioperative myocardial infarction and early graft occlusion after coronary artery bypass surgery. Chest. 2002;121: 103-111.

7. Levitski S, Sidney MD. Protecting the myocardial cell during coronary revascularization. The Willian W.L. Glenn Lecture. Circulation. 2006;114:I-339-43.

8. Slogoff S, Stephen M. D, Keats A, Arthur S. Does perioperative ischemia lead to postoperative myocardial infarction?. Anesthesiology. 1985;62:107-14.

9. Force T, Hibberd P, Weeks G, Kemper AJ, Bloomfield P, Tow D, et al. Perioperative myocardial infarction after coronary artery bypass surgery. Clinical significance and approach to risk stratification. Circulation. 1990;82:903-12.

10. Onorati F, De Feo M, Mastroroberto P, Cristodoro L, Pezzo F, Renzulli A, et al. Determinants and prognosis of myocardial damage after coronary artery bypass grafting. Ann Thorac Surg. 2005;79:837-45.

11. Slogoff $\mathrm{S}$, Keats AS. Myocardial ischemia revisited. Anesthesiology. 2006;105:214-16.

12. Greaves SC, Rutherford JD, Aranki SF, Cohn LH, Coupper GS, Adams $\mathrm{DH}$, et al. Current incidence and determinants of perioperative myocardial infarction in coronary artery surgery. Am Heart J. 1996;132:572-8.

13. Chaitman BR, Alderman EL, Sheffield LT, Tong T, Fisher L, Mock $M B$. Use of survival analysis to determine the clinical significance of new $Q$ waves after coronary bypass surgery. Circulation. 1983;63:302.

14. Thielmann $M$, Massoudy $P$, Neuhauser $M$, Knipp $S$, Kamler $M$, Piotrowski $\mathrm{J}$, et al. Prognosis value of preoperative cardiac troponin I in patients with non-ST-segment. Elevation acute coronary syndromes undergoing coronary artery bypass surgery. Chest. 2005;128:3526-36.

15. Thielmann M, Massoudy $P$, Jaeger BR, Neuhäuser M, Marggraf $G$, Sack $S$, et al. Emergency re-revascularization with percutaneous coronary intervention, reoperation, or conservative treatment in patients with acute perioperative graft failure following coronary artery bypass surgery. Eur J Cardio-Thorac Surg. 2006;30:117-25.

16. Podgoreanu MV, White W. D, Morris R. W, Mathew J. P, StaffordSmith M, Welsby I. J. Inflamatory gene polimorphisms and risk of postoperative myocardial infarction after cardiac surgery. Circulation. 2006;114:I-275-81.

17. Yau TM. Predictors operative risk for coronary bypass operations in patients with left ventricular disfunction. J Thorac Surg. 2005;79:837-45.

18. Chanda J, Brichkov I, Canver CC. Prevention of radial artery graft vasospasm after coronary bypass. Ann Thorac Surg. 2000;70:2070-4.

19. Alter $P$, Vogt $S$, Herzum $M$, Irqusi $M$, Rupp $H$, Maisch $B$, et al. Indications for angiography subsequent to coronary artery bypass grafting. Am Heart J. 2005;149:1082-90.

20. Januzzi JL, Lewandrowski K, MacGillivray TE, Newell JB, Kathiresan S, Servess SJ, et al. A comparison of cardia troponin $\mathrm{T}$ and creatin kinase-mb for patients evaluation after cardiac surgery. J Am Coll Cardiol. 2002;39:1518.

21. Leong DK, Ashok V, Nishkantha A, Shau YH, Sim EK. Transit- time flow measurement is essential in coronary artery bypass grafting. Ann Thorac Surg. 2005;79:854-8.

22. González Santos JM, López Rodríguez J, Dalmau MJ. Los injertos arteriales en cirugía coronaria: ¿una terapia universal?. Rev Esp Cardiol. 2005;58:1207-23.

23. Colmenero Ruiz M, García Delgado M, Pérez Villares JM, Ramírez Hernández JA, Reina Toral A. Vasoespasmo de los injertos coronarios en el postoperatorio de cirugía de revascularización miocárdica. Med Intensiva. 2001;25:127-30.

24. Michael G, Fitzsimons MD, Walker J, Inglessis I, Boucher C. Left main coronary artery spasm: Medical versus surgical management. J Cardiothorac Vasc Anesth. 2006;20:834-6.

25. Lin CY, Weng ZC, Loh SH, Hong GJ, Tsai CS. Coronary artery spasm after off-pump coronary artery bypass grafting. ANZ J Surgery. 2007;77:126-9.

26. Santaló Bela M, Guindo Soldevilab J, Ordoñez Llanos J. Marcadores biológicos de necrosis miocárdica. Rev Esp Cardiol. 2003;56:703-20.

27. Sadony V, Korber M, Albes G, Podtschaske V, Etgen T, Trosken T, et al. Cardiac troponin I plasma levels for diagnosis and quantitation of perioperative myocardial damage in patients undergoing coronary artery bypass surgery. Eur J Cardiothorac Surg. 1998;13:57-65. 
28. UptoDate. Troponins, creatine kinase and CPK isoforms as biomarkers of cardiac injury [citado 5 Sep 2009]. Disponible en: URL: http://uptodate.papi.h12o.es/online/content/topic.do? topickey=chd/

13852\&selectedTitle=1 150\&source=search_result.

29. Van Lente F, Marin A, Ratliff NB. The predictive value of serum enzimes for perioperative myocardial infarction after cardiac operations. An autopsy study. J Thorac Cardiovasc Surg. 1989;98:704-10.

30. Carrier M, Pellerin M, Perrault LP, Solymoss BC, Pelletier LC. Troponin I levels in patients with myocardial infarction after coronary artery bypass grafting. Ann Thorac Surg. 2000;69: $435-440$.

31. Benoit MO, Paris M, Silleran J, Fiemeyer A, Moatti N. Cardiac troponin I: Its contribution to the diagnosis of perioperative myocardial infarction and various complications of cardiac surgery. Critical Care Med. 2001;29:1880-6.

32. Fransen EJ, Diris JH, Maessen JG, Hermens WT, Van DieijenVisser MP. Evaluation of "New" cardiac markers for ruling out myocardial infarction after coronary artery bypass grafting. Chest. 2002;122:1316-21.

33. Peivandi AA, Dahm M, Opfermann VT, Peetz D, Doerr F, Loos A, et al. Comparison of cardiac troponin I versus $T$ and creatine kinase $\mathrm{Mb}$ after coronary artery bypass grafting in patients with and without perioperative myocardial infartion. Hertz. 2004;29:658-64.

34. Tzimas PG, Milionis HJ, Arnaoutoglou HM, Kalantzi KJ, Pappas K, Karfis E, et al. Cardiac troponin I versus creatine kinase-Mb in the detection of postoperative cardiac events after coronary artery bypass grafting surgery. J Cardiovasc Surg. 2008;49: 95-101.

35. Nesher N, Alghamdi AA, Singh SK, Sever JY, Christakis GT, Goldman BS, et al. Troponin after cardiac surgery: A predictor or a phenomenon?. Ann Thorac Surg. 2008;85:1348-54.

36. Noora J, Ricci C, Hastings D, Hill S, Cybulsky I. Determination of troponin I release after CABG Surgery. J Card Surg. 2005;20: $129-135$.

37. Thielmam M, Massoudy P, Marggraf G, Knipp S, Schmermund A, Piotrowski J, et al. Role of troponin I, myoglobin and creatine kinase for the detection of early graft failure following coronary artery bypass grafting. Eur J Cardiothorac Surg. 2004;26:102-9.

38. Alyanakian MA, Dehoux M, Chatel D, Seguret C, Desmonts JM, Durand G, et al. Cardiac troponin I in diagnosis of perioperative myocardial infarction after cardiac surgery. J Cardiothorac Vasc Anesth. 1998;12:288-94.

39. Gensini GF, Fusi C, Conti AA, Calamai GC, Montesi GF, Galanti G, et al. Cardiac troponin I and Q-wave perioperative myocardial infarction after coronary artery bypass surgery. Crit Care Med. 1998;26:1986-90.

40. Castro MJ, Vázquez Rizaldos S, Velayos Amo C, Herranz Valera J, Almeria Valera C, Iloro Mora MI. Cardiac troponin I in perioperative myocardial infarction after coronary artery bypass surgery. Rev Esp Cardiol. 2002;55:245-50.

41. Botha P, Nagarajan DV, Lewis PS, Dunning J. Can cardiac troponins be used to diagnose a perioperative myocardial infarction post cardiac surgery?. Interact Cardiovasc Thorac Surg. 2004;3:442-9.

42. Bruce Martin C, Shaw AD, Janos G, Aravindan N, Murphy F, Royston $D$, et al. The comparison and validity of troponin I assay systems in diagnosing myocardial ischemic injury after surgical coronary revascularization. J Cardiothorac Vasc Anesth. 2005;19:288-93.

43. Thygesen K, Alpert JS, White HD. Task force for the redefinition of myocardial Infarction. J Am Coll Cardiol. 2007;50:2173-95.
44. Myocardial infarction redefined-a consensus document of the Joint European Society of Cardiology/American College of Cardiology Committee for the redefinition of myocardial infarction. Eur Heart J. 2000;21:1502-13.

45. Wang Asinger RW, Marriott HJ. ST-segment elevation in conditions other than acute myocardial infartion. $\mathrm{N}$ Engl Med. 2003;349:2128-35.

46. Uday J, Laflamme Claude JA, Aggarwal A, Ramsay James G, Comunale Mark E, Ghoshal S, et al. Electrocardiographic and hemodinamyc changes and their association with myocardial infarction during coronary artery bypass surgery: A multicenter study. Anesthesiology. 1997;86:576-91.

47. Rasmussen C, Thiis J, Clemmensen P, Efsen F, Arendrup H, Saunamaki K, et al. Significance and management of early graft failure after coronary artery bypass grafting: Feasability and results of acute angiography and re-re-vascularization. Eur $\mathrm{J}$ Cardiothorac Surg. 1997;12:847-52.

48. Fabricius M, Gerber W, Hanke M, Garbade J, Autschbach R, Mohr FW. Early angiographyc control of perioperative ischemia after coronary artery bypass grafting. Eur J Card Thor Surg. 2001;19:853-8.

49. Sánchez F, García JM, Aragonés R, Delgado M, Matas MD, Vera A. La troponina I como predictor de morbilidad tras cirugía cardíaca con circulación extracorpórea. Med Intensiva. 2001;25:257-62.

50. Fernández Del Campo R, Martínez Lozano F, Aldániz Echevarria G, Martínez Nuñez JJ, Viven Bonato GG, González Prendes C. Postoperatorio de cirugía cardíaca y vascular. Complicaciones más frecuentes en el postoperatorio de cirugía cardíaca. Med Intensiva. 2002;26:155-60.

51. Klate K, Chaitman BR, Theroux P, Gavard JA, Stocke k, Boyce S, et al. Increased mortality after coronary artery bypass graft surgery in associated with incrased levels of postoperative creatine kinase myocardial band isoenzime release: Results from the Guardian trial. J Am Coll Cardiol. 2001;38:1070.

52. Hirotani T, Kadema T, Shirota S, Y. Nakao. An evaluation of the intraoperative transit time meaurements of coronary bypass flow. Eur J Cardiothorac Surg. 2001;19:848-52.

53. Taggart DP, Choudhary B, Anastasiadis K, Abu-Omar Y, Balacumaraswami L, Pigott DW. Preliminary experience with novel intraoperative fluorescence imaging technique to evaluate the patency of bypass grafts in total arterial revascularization. Ann Thorac Surg. 2003;75:870-3.

54. Nimesh D, Miwa S, Kodama D, Cohen G, Christakis GT, Cohen G, et al. Improving the quality of coronary bypass surgery with intraoperative angiography. J Am Coll Cardiol. 2005;46:1521-5.

55. Di Giammarco G, Pano M, Cirmeni S, Pelini P, Vitolla G, Di Marco $M$. Predictive value of intraoperative transit-time flow measurement for short-term graft patency in coronary surgery. $J$ Thorac Cardiovasc Surg. 2006;132:468-74.

56. Hol PK, Lingaas PS, Lundbland R, Rein KA, Vatne K, Smith HJ, et al. Intraoperative angiography leads to graft revision in coronary artery bypass surgery. Ann Thorac Surg. 2004;78: 502-505.

57. KuliK A, Rubens FD, Ruel M. Intraoperative indocyanine green angiography: Ready for prime time?. J Thorac Cardiovasc Surg. 2007;133:592-3.

58. Smith SC Jr, Feldman TE, Hirshfeld JW Jr, Jacobs AK, Kern MJ, King SB 3rd, et al. ACC/AHA/SCAl 2005 guideline update for percutaneus coronary intervention a report of the American College of Cardiology/American Heart Association task force on practice guidelines ACC/AHA/SCAl. Writing to update the 2001 guidelines for percutaneous coronary intervention. J Am Coll Cardiol. 2006;47:e1. 